\title{
INVARIANT SUBSPACES OF FINITE CODIMENSION FOR MEASURES WITH THIN SUPPORT
}

\author{
T. T. TRENT
}

(Communicated by Paul S. Muhly)

\begin{abstract}
A simple proof that $M_{z}$ on $P^{2}(\mu)$ has a nontrivial invariant subspace is given. If $P^{2}(\mu) \neq L^{2}(\mu)$ and if $\mu$ has "thin" support, then $P^{2}(\mu)$ has bounded point evaluations.
\end{abstract}

In Thomson [T] a simple proof that subnormal operators have nontrivial invariant subspaces was given. An extension of this result to certain algebras of subnormal operators was provided in Trent [Tr] and Trent-Wogen [TW] and, also, in Yan [Y]. In this paper we give a streamlined version of Trent [Tr], which gives invariant subspaces of subnormal operators and, in addition, in the case of measures with "thin" support, gives subspaces of finite codimension (in the nonnormal case). Thus for such measures we have the existence of bounded point evaluations.

Let $\mu$ denote a positive compactly supported Borel measure in the complex plane $\mathbf{C}$. By $P^{2}(\mu)$ we mean the closure of the analytic polynomials in $L^{2}(\mu)$. A well-known result of Bram [B] reduces the invariant subspace problem for subnormal operators to the problem for the cyclic subnormal operator, multiplication by $z, M_{z}$, on $P^{2}(\mu)$. (See Conway [C] for the terminology.) First we give a complete proof that $M_{z}$ on $P^{2}(\mu)$ has a nontrivial invariant subspace when $\mu$ is not a multiple of a point mass measure.

In the case that $P^{2}(\mu)=L^{2}(\mu)$, then for any Borel set $E, \chi_{E} L^{2}(\mu)$ is an invariant subspace for $M_{z}$. If $\mu$ is not a multiple of a point mass measure, then $E$ can be chosen so that $\{0\} \$ \chi_{E} L^{2}(\mu) \$ L^{2}(\mu)$, so $\chi_{E} L^{2}(\mu)$ is a nontrivial invariant subspace for $M_{z}$. If $\mu$ is a multiple of a point mass, then $L^{2}(\mu)$ $\left(=P^{2}(\mu)\right)$ is a one dimensional Hilbert space. This case is easily disposed of, so we assume that the support of $\mu$ contains more than one point.

Received by the editors June 23, 1989.

1980 Mathematics Subject Classification (1985 Revision). Primary 47B20, 47A15, 46E99.

Key words and phrases. Subnormal operator, invariant subspace, bounded point evaluation.

Most of this work was done while the author enjoyed the hospitality of the Mathematics Department of Indiana University at Bloomington, Indiana.

The author was partially supported by a grant from the Research Grants Committee of the University of Alabama. 
From the above discussion, to find a nontrivial invariant subspace for $M_{z}$ on $P^{2}(\mu)$, we may assume $P^{2}(\mu) \neq L^{2}(\mu)$. In this case it is an open problem whether evaluation at a point induces a bounded linear operator on $P^{2}(\mu)$. (See Brennan [Br].) Using the Riesz representation theorem this is easily seen to be equivalent to the problem of the existence of a nontrivial eigenvector for $M_{z}^{*}$. But $M_{z}^{*}$ has a nontrivial eigenvector if and only if $M_{z}$ has a nontrivial invariant subspace of finite codimension.

We will show that if $P^{2}(\mu) \neq L^{2}(\mu)$ and if the linear manifold consisting of analytic polynomials plus conjugates of analytic polynomials is $\omega^{*}$-dense in $L^{\infty}(\mu)$, then $P^{2}(\mu)$ has bounded point evaluations. This condition is satisfied, for example, when the support of $\mu$ is carried by the outer boundary of a compact set. Of course more abstractly defined measures may also have this property. A necessary condition for this density property is that the interior of the support of $\mu$ be empty. Thus we refer to measures having this $\omega^{*}$-density property as measures of "thin" support.

Theorem $\mathbf{1}$ (Brown [Bro]). $M_{z}$ on $P^{2}(\mu)$ has a nontrivial invariant subspace.

Proof. By the previous discussion we may assume that $P^{2}(\mu) \neq L^{2}(\mu)$. Choose $0 \neq h \in L^{2}(\mu)$ with $h \perp P^{2}(\mu)$ and $\lambda \in \mathbf{C}$ such that

$$
\int\left|\frac{h(z)}{z-\lambda}\right|^{3 / 2} d \mu(z)<\infty
$$

and

$$
A_{\lambda} \triangleq \int \frac{\overline{h(z)}}{z-\lambda} d \mu(z) \neq 0 .
$$

(We will sketch the proof later that such an $h$ and $\lambda$ actually exist.) Define

$$
\omega(z)=\left(1+\frac{|h(z)|}{|z-\lambda|}\right)^{1 / 2} \quad \text { for } \mu \text {-a.e. } z \text { in } \mathbf{C}
$$

and note that $\omega(z) \geq 1$. Now since $h \perp P^{2}(\mu)$,

$$
0=\int \frac{p(z)-p(\lambda)}{z-\lambda} \overline{h(z)} d \mu(z) \text {. }
$$

So

$$
\begin{aligned}
|p(\lambda)| & \leq \frac{1}{\left|A_{\lambda}\right|} \int|p(z)|\left|\frac{h(z)}{z-\lambda}\right| d \mu(z) \\
& \leq \frac{1}{\left|A_{\lambda}\right|} \int|p(z)| \omega(z) \omega(z) d \mu(z) .
\end{aligned}
$$

Use the Cauchy-Schwartz inequality on the functions $|p|$ and $\omega$ with respect to the measure $\omega d \mu$ to get

$$
|p(\lambda)| \leq \frac{1}{\left|A_{\lambda}\right|}\|p\|_{2, \omega d \mu}\left(\int \omega^{3} d \mu\right)^{1 / 2} .
$$


By (1) evaluation at $\lambda$ is a bounded linear functional on $P^{2}(\omega d \mu)$. So by the Riesz representation theorem there exists a $k_{\lambda} \in P^{2}(\omega d \mu)$ such that

$$
p(\lambda)=\left\langle p, k_{\lambda}\right\rangle_{\omega d \mu} .
$$

Since $\omega \geq 1, P^{2}(\omega d \mu) \subset P^{2}(\mu)$; hence, $k_{\lambda} \in P^{2}(\mu)$. Let

$$
\mathscr{M}=\left\{(z-\lambda) p(z) k_{\lambda}(z): p \text { a polynomial }\right\}^{-L^{2}(\mu)} \text {. }
$$

If $\mathscr{M}=\{0\}$, then $k_{\lambda}=C \chi_{\{\lambda\}} \neq 0$ in $P^{2}(\mu)$. But then $\chi_{\mathrm{C}-\{\lambda\}} P^{2}(\mu)$ is a nontrivial invariant subspace (of finite codimension) for $\mathscr{M}_{z}$ on $P^{2}(\mu)$. Else $\mathscr{M} \neq\{0\}$. Now $\omega k_{\lambda} / \bar{k}_{\lambda} \in L^{2}(\mu)$ by (1) (where $k_{\lambda} / \bar{k}_{\lambda}$ is set equal to 0 if $\left.k_{\lambda}(z)=0\right)$ and

$$
\left\langle p k_{\lambda}, \omega k_{\lambda} / \bar{k}_{\lambda}\right\rangle_{\mu}=\left\langle p, k_{\lambda}\right\rangle_{\omega d \mu}=p(\lambda) .
$$

Thus $\omega k_{\lambda} / \bar{k}_{\lambda} \perp \mathscr{M}$, but $\omega k_{\lambda} / \bar{k}_{\lambda} \not k_{\lambda} \in P^{2}(\mu)$. Hence $M \supsetneqq P^{2}(\mu)$, which completes the proof.

Theorem 2. Assume that $P^{2}(\mu) \neq L^{2}(\mu)$ and that $\mu$ has "thin" support. Then $P^{2}(\mu)$ has a bounded point evaluation.

Proof. By the proof of Theorem 1 we need only consider the case where $\{0\} \supsetneqq$ $\mathscr{M} \supsetneqq P^{2}(\mu)$. ( $\mathscr{M}$ is defined in the proof of Theorem 1.) By our introductory remarks it suffices to show that $\mathscr{M}$ has finite codimension in $P^{2}(\mu)$. Clearly, if $k_{\lambda}$ is a cyclic vector for $\mathscr{M}_{z}$ on $P^{2}(\mu)$ then $\mathscr{M}=\left\{(z-\lambda) P^{2}(\mu)\right\}^{-}$and we're done. Since $\omega \geq 1$ it is sufficient to show that $k_{\lambda}$ is a cyclic vector for $\mathscr{M}_{z}$ on $P^{2}(\omega d \mu)$.

We need a further reduction. If $\mathscr{M}_{z}$ is reducible on $P^{2}(\omega d \mu)$, then there exists a nontrivial projection $P$ commuting with $M_{z}$. But a theorem of Yoshino [Yo] says that $P$ is just multiplication by $\chi_{E}$ for some Borel set $E$ and $\chi_{E} \in L^{\infty}(\mu) \cap P^{2}(\omega d \mu) \subset L^{\infty}(\mu) \cap P^{2}(\mu)$. Hence $\mathscr{M}_{z}$ is reducible on $P^{2}(\mu)$. It follows from a result of Conway [C, p. 296], based on theorems of Behncke and of Berger and Shaw, that

$$
P^{2}(\mu)=L^{2}\left(\left.\mu\right|_{\Delta_{0}}\right) \oplus \sum^{\oplus} P^{2}\left(\left.\mu\right|_{\Delta_{i}}\right)
$$

where $\left\{\Delta_{i}\right\}_{i=1}^{\infty}$ is a collection of pairwise disjoint Borel sets. For the above decomposition $L^{2}\left(\left.\mu\right|_{\Delta_{i}}\right)$ and $P^{2}\left(\left.\mu\right|_{\Delta_{i}}\right)$ reduce $\mathscr{M}_{z}$ on $P^{2}(\mu)$. Furthermore, $\mathscr{M}_{z} \mid P^{2}\left(\left.\mu\right|_{\Delta_{i}}\right)$ is irreducible. If $P^{2}(\mu) \neq L^{2}(\mu)$, then for some $i, P^{2}\left(\left.\mu\right|_{\Delta_{i}}\right) \neq$ $L^{2}\left(\left.\mu\right|_{\Delta_{i}}\right)$ and clearly from our hypothesis of $\mu$ with "thin" support we have $\left.\mu\right|_{\Delta_{i}}$ has "thin" support. Thus without loss of generality we may assume that $\mathscr{M}_{z}$ on $P^{2}(\mu)$ is irreducible.

We assume that $\mathscr{M}_{z}$ on $P^{2}(\mu)$ is irreducible and that $\mu$ has "thin" support, and show that $k_{\lambda}$ is cyclic for $\mathscr{M}_{z}$ on $P^{2}(\omega d \mu)$ (and thus cyclic for $P^{2}(\mu)$ ). For ease of notation let $\lambda=0$. 
Assume that $k_{0}$ is not cyclic for $\mathscr{M}_{z}$ on $P^{2}(\omega d \mu)$. Then there exists $0 \neq$ $F \in P^{2}(\omega d \mu)$ and $F \perp p k_{0}$ for all polynomials $p$. Thus

$$
0=\int \bar{z}^{n} F \bar{k}_{0} \omega d \mu, \quad n \geq 0 .
$$

But since $k_{0}$ is evaluation at 0 ,

$$
0=0^{n} F(0)=\int z^{n} F \bar{k}_{0} \omega d \mu, \quad n \geq 1
$$

(where $F(0) \triangleq\left\langle F, k_{0}\right\rangle_{\omega d \mu}$ ). Thus

$$
0=\int(p+\bar{q}) F \bar{k}_{0} \omega d \mu \quad \text { for all polynomials } p \text { and } q .
$$

Since $\omega d \mu$ has "thin" support,

$$
0=F \bar{k}_{0} \omega \quad \mu \text {-a.e. }
$$

and since $\omega \geq 1$

$$
0=F \bar{k}_{0} \quad \mu \text {-a.e. }
$$

We see that

$$
0=\int z^{n} k_{0} \bar{z}^{m} F d \mu \quad \text { for all } n, m \geq 0 \text { and }
$$

all $F \perp\left\{p k_{0}: p\right.$ a polynomial $\}$.

This shows that $\mathscr{M}_{z}$ is reducible, a contradiction.

We note that while our proof actually leads to a set of $\lambda$ 's of positive area measure which induce bounded point evaluations, it does not seem to give the existence of an open set of bounded point evaluations.

It would be of interest to weaken the $\omega^{*}$-density hypothesis to

$$
\{p+\bar{q}: p, q \text { polynomials }\}^{-L^{2}(\mu)}
$$

and conclude that bounded point evaluations exist. We have been unable to do this. It should be noted, however, that a modification of our proof shows that, if $P^{2}(\mu) \neq L^{2}(\mu)$ and if, for $j$ fixed,

$$
\left\{p_{1}+\bar{p}_{2}+z \bar{p}_{3}+\cdots+z^{j} \bar{p}_{j}: p_{i} \text { a polynomial }\right\}^{-\omega^{*}}=L^{\infty}(\mu),
$$

then we may again conclude that $P^{2}(\mu)$ has bounded point evaluations. Now for any measure $\mu$, the Stone-Weierstrass theorem tells us that

$$
\left\{p+\sum_{l=0}^{j} z^{l} \bar{p}_{l}: p, p_{l} \text { are polynomials and } j=0,1,2, \ldots\right\}^{-\omega^{*}}=L^{\infty}(\mu) .
$$

Hence the general existence problem for bounded point evaluations corresponds to the case when $j$ is unrestricted. 
For completeness we sketch the proofs of (1) and (2) of Theorem 1. Let $K$ denote the support of the measure $\mu$, let $\Delta_{R}(a)$ denote a disc of radius $R$ centered at $a$, and let $m_{2}$ denote area Lebesgue measure on $\mathbf{C}$.

Proof of (1). Assume that $K \subset \Delta_{R}(0)$. Consider

$$
(*)=\int_{\Delta_{R(0)}} \int_{K}\left|\frac{h(z)}{z-\lambda}\right|^{3 / 2} d \mu(z) d m_{2}(\lambda) .
$$

By Fubini's theorem,

$$
\begin{aligned}
(*) & =\int_{K} \int_{\Delta_{R(0)}} \frac{1}{|z-\lambda|^{3 / 2}} d m_{2}(\lambda)|h|^{3 / 2} d \mu \\
& \leq \int_{K} \int_{\Delta_{2 R(z)}}\left(\frac{1}{r}\right)^{3 / 2} r d r d \theta|h|^{3 / 2} d \mu \\
& \leq 2(2 R)^{1 / 2} \int_{K}|h|^{3 / 2} d \mu<\infty \quad \text { since } h \in L^{2}(\mu) .
\end{aligned}
$$

It is easy to see that (1) follows.

Proof of (2). (2) is usually established using Green's theorem to give an integral representation of a $C^{1}$ function. We give an alternative proof. Assume that $P^{2}(\mu) \neq L^{2}(\mu)$ and that (2) fails. Then for all $\phi \perp P^{2}(\mu)$ and for $m_{2}$-a.e. $\lambda \in \mathbf{C}$

$$
0=\int_{K} \frac{\overline{\phi(z)}}{z-\lambda} d \mu(z)
$$

Then, for all $a \in \mathbf{C}$ and $R>0$,

$$
\begin{aligned}
& 0=\int_{\Delta_{R(a)}}(a-\lambda) \int_{K} \frac{\overline{\phi(z)}}{z-\lambda} d \mu(z) d m_{2}(\lambda), \\
& 0=\int_{K} \overline{\phi(z)} \int_{\Delta_{R(a)}} \frac{(a-\lambda)}{z-\lambda} d m_{2}(\lambda) d \mu(z)
\end{aligned}
$$

by Fubini's theorem and (1).

$$
\begin{aligned}
0 & =\int_{K} \overline{\phi(z)}\left[2 \pi \int_{|a-z|}^{R} r d r\right] d \mu(z), \quad \text { using polar coordinates. } \\
0 & =\int_{K} \overline{\phi(z)}\left[R^{2}-\left(|a|^{2}-z \bar{a}-\bar{z} a+|z|^{2}\right) d \mu(z) .\right.
\end{aligned}
$$

But $\phi \perp 1, \phi \perp z$ and letting $a=0$ gives $0=\int_{K} \bar{\phi}|z|^{2} d m_{2}(z)=0$. Now let $a=1$ to get

$$
0=\int_{K} \bar{\phi} \bar{z} d \mu(z), \quad \text { i.e., } \phi \perp \bar{z} .
$$

Since this is true for all $\phi \perp P^{2}(\mu)$, we must have $\bar{z} \in P^{2}(\mu)$. So polynomials in $z$ approximate $\bar{z}$ in $L^{2}(\mu)$. It is not hard to see that $z^{n} \bar{z}^{m} \in P^{2}(\mu)$ for 
$m, n=0,1,2, \ldots$. Thus by the Stone-Weierstrass theorem $P^{2}(\mu)=L^{2}(\mu)$, a contradiction.

Addendum. Since this paper was submitted, we have received a preprint from J. Thomson which totally solves the existence problem for b.p.e.'s.

\section{REFERENCES}

[B] J. Bram, Subnormal operators, Duke Math. J. 22 (1955), 75-94.

[Br] J. Brennan, Point evaluations, invariant subspaces, and approximation in the mean by polynomials, J. Funct. Anal. 34 (1979), 407-420.

[Bn] S. Brown, Some invariant subspaces for subnormal operators, Integral Equations Operator Theory 1 (1978), 310-333.

[C] J. Conway, Subnormal operators, Pitman, Boston, 1981.

[T] J. Thomson, Invariant subspaces for algebras of subnormal operators, Proc. Amer. Math. Soc. 96 (1986), 462-464.

[Tr] T. T. Trent, Invariant subspaces for operators in subalgebras of $L^{\infty}(\mu)$, Proc. Amer. Math. Soc. 99 (1987), 268-272.

[TW] T. Trent and W. Wogen, Subnormal operators with a common invariant subspace, preprint.

[Y] K. Yan, Invariant subspaces for joint subnormal systems, preprint.

[Yo] T. Yoshino, Subnormal operators with cyclic vector, Tohoku Math. J. 25 (1969), 47-55.

Department of Mathematics, University of Alabama, Tuscaloosa, Alabama 35487 